\title{
Active Mode Estimation for Switching Systems
}

\author{
Elom Ayih Domlan, José Ragot and Didier Maquin \\ Institut National Polytechnique de Lorraine \\ Centre de Recherche en Automatique de Nancy, UMR 7039 CNRS-UHP-INPL \\ 2, avenue de la forêt de Haye \\ 54516 Vandœuvre-lès-Nancy, France \\ \{elom-ayih.domlan, jose.ragot, didier.maquin\}@ensem.inpl-nancy.fr
}

\begin{abstract}
Switching systems are a particular class of hybrid systems. They are described by several operating regimes, called modes, each of them being active under certain particular conditions. When the switching mechanism is perfectly known, it is easy to handle such systems because the knowledge of the active regime at any moment is available. On the other hand, in the case where there is no information about the switching mechanism, the situation is more complicated. In this configuration, it is difficult to carry out a fault diagnosis scheme or to synthesize a control law. This paper addresses the issue of the determination of the active mode at any moment, using only the system's input/output data. Conditions that guarantee the uniqueness of the determined active mode are also given.
\end{abstract}

\section{INTRODUCTION}

The modeling of complex systems often leads to complex non-linear models. To get rid of the obtained model's complexity, a widely used modeling strategy is to represent the system's behavior using a set of models with simple structure, each model describing the behavior of the system in a particular operating zone. Within this modeling framework, hybrid models are very successful in representing such processes.

Hybrid models [1], [2] characterize physical processes governed by continuous differential equations and discrete variables. The process is described by several operating regimes, called modes, and the transition from one mode to another is governed by a mechanism which depends on the system's variables (input, output, state) or external variables (human operator for instance).

The global behavior obtained for the modeled complex system is strongly related to the nature of the procedure managing the transition from one mode to another. When the transition from one mode to another is abrupt, one obtains a particular, but significant, class of systems namely switching models. This class of models is widely used because tools for analysis and control of linear systems are well mastered and, moreover, many real processes can be represented by models belonging to this class.

Research on switching systems is mainly focused in the fields of identification [2]-[5], control [6], stability analysis [7] and state estimation [8], [9]. The knowledge of the mode describing the evolution of the system at any moment, this mode being called active mode, is a crucial information that simplifies the application of the results coming from the fields of identification, control, stability analysis and state estimation. This paper presents a method for the determination of the active mode of a switching system at any instant using the measurement data (input and output) of the system.

Section II introduces the retained model for the representation of switching systems and states the problem under investigation. The results for the determination of the active mode are presented in section III. Conditions guaranteeing the discernability of the various modes are then formulated in section IV. In order to illustrate the proposed methods, an academic example is shown in section VI .

\section{Position OF THE PROBlem}

Let us consider the system represented by equation (1):

$$
\begin{aligned}
& \left\{\begin{array}{l}
x(k+1)=A_{\mu_{k}} x(k)+B u(k) \\
y(k)=C x(k)
\end{array}\right. \\
& x \in \mathbb{R}^{n}, u \in \mathbb{R}^{m}, y \in \mathbb{R}^{p} \\
& \mu_{k} \in\{1,2, \ldots, s\}, s \in \mathbb{N}^{*} \backslash\{1\}
\end{aligned}
$$

Equation (1) represents a switching system with $s$ operating regimes or modes. The variables $u(\cdot), y(\cdot)$ and $x(\cdot)$ respectively stand for the input, the output and the state of the system. The switchings are introduced by means of the system's state matrix which takes its value in a finite set $A=\left\{A_{1}, A_{2}, \ldots, A_{s}\right\}$ which is a priori known. This formulation does not restrict at all the modeling of the switching system and the results presented in this paper can be extended to the case where the matrices $B$ and $C$ also take different values. The variable $\mu_{(\cdot)}$ denotes the active mode at any moment. For example, if one has $\mu_{k}=i$, $i \in\{1,2, \ldots, s\}$, the system is said to be in the mode $i$ at the instant $k$. We assume that the switchings are triggered by unknown external variables and then, the mode sequence is arbitrary and independent of the system's variable (input, output and state).

Coming from (1), we wish to recover the active mode (or the value taken by $\left.\mu_{(\cdot)}\right)$ at any moment, using only the system's input/output data on a finite observation window. We introduce the following definitions:

Definition 1 (Path): A path $\mu$ is a finite sequence of modes: $\mu=\left(\mu_{1} \cdot \mu_{2} \cdot \ldots \cdot \mu_{h}\right)$. The length of a path $\mu$ is denoted $|\mu|$ and for $\mu=\left(\mu_{1} \cdot \mu_{2} \cdot \ldots \cdot \mu_{h}\right)$, one has $|\mu|=h$. The set of all paths of length $h$ is denoted $\Theta_{h}$. 
$\mu_{[i, j]}$ is the infix of the path $\mu$ between $i$ and $j: \mu_{[i, j]}=$ $\left(\mu_{i} \cdot \mu_{i+1} \cdot \ldots \cdot \mu_{j}\right)$.

Definition 2 (Observability matrix): The observability matrix $\mathcal{O}_{\mu, h}$ of a path $\mu \in \Theta_{h}$ is defined as :

$$
\mathcal{O}_{\mu, h}=\left(\begin{array}{c}
C \\
C A_{\mu_{1}} \\
\vdots \\
C \underbrace{A_{\mu_{h-1}} A_{\mu_{h-2}} \cdots A_{\mu_{1}}}_{h-1}
\end{array}\right)
$$

In order to provide more explanation about definitions (1) and (2), let us consider again the model of equation (1) with $s=2$ (two modes). Table I shows the set $\Theta_{3}$ of all paths of length 3 for this system.

TABLE I

SET OF ALL PATHS OF LENGTH 3

\begin{tabular}{lcccccccc}
\hline Path & $\mu^{1}$ & $\mu^{2}$ & $\mu^{3}$ & $\mu^{4}$ & $\mu^{5}$ & $\mu^{6}$ & $\mu^{7}$ & $\mu^{8}$ \\
\hline$\mu_{k-3}$ & 1 & 1 & 1 & 1 & 2 & 2 & 2 & 2 \\
$\mu_{k-2}$ & 1 & 2 & 1 & 2 & 1 & 2 & 1 & 2 \\
$\mu_{k-1}$ & 1 & 1 & 2 & 2 & 1 & 1 & 2 & 2 \\
\hline
\end{tabular}

The path $\mu^{4}$ (fifth column of table I) describes a mode sequence in which the system is in mode 1 at the time $k-3$, then switches into mode 2 at $k-2$ and stays in mode 2 at the instant $k-1$. In this configuration, the corresponding path is $\mu^{4}=(1 \cdot 2 \cdot 2)$. For the path $\mu^{4}=(1 \cdot 2 \cdot 2)$, the observability matrices is defined as:

$$
O_{\mu^{4}, h}=\left(\begin{array}{c}
C \\
C A_{1} \\
C A_{2} A_{1} \\
C A_{2} A_{2} A_{1}
\end{array}\right)
$$

Definition 3 (Active path): On a finite observation window $[k-h, k]$, the active path $\mu^{*}$ is the one describing the true mode sequence on the observation window.

From definitions 1 and 3, the estimation of the active mode at any moment is equivalent to the determination of the path describing the true mode sequence on a finite observation window. For that, throughout the remainder of this paper, we will focus on the recovery of the active path on an observation window.

\section{ACTIVE PATH DETERMINATION}

The determination of the active path can be formulated as a recursive problem applied to a sliding window. On a time window $[k-h, k]$, equation (1) can be written as:

$$
\mathcal{O}_{\mu, h} x(k-h)=\left(\begin{array}{c}
y(k) \\
\vdots \\
y(k-h)
\end{array}\right)-\mathcal{T}_{\mu, h}\left(\begin{array}{c}
u(k) \\
\vdots \\
u(k-h)
\end{array}\right)
$$

where $\mathcal{T}_{\mu, h}$ is a Toeplitz matrix defined by :

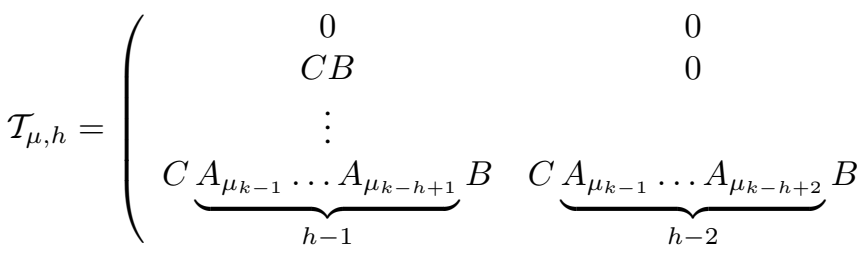

$$
\begin{aligned}
& \left.\begin{array}{ccc}
\ldots & 0 & 0 \\
& 0 & 0 \\
& \vdots & \vdots \\
\ldots & C B & 0
\end{array}\right)
\end{aligned}
$$

Equation (4) can be written in a more compact way:

$$
Y_{k-h, k}-\mathcal{T}_{\mu, h} U_{k-h, k}=\mathcal{O}_{\mu, h} x(k-h)
$$

where $\left.Y_{k-h, k}=\left(\begin{array}{llll}y(k) & y(k-1) & \ldots & y(k-h\end{array}\right)\right)^{T}$ and $U_{k-h, k}=\left(\begin{array}{llll}u(k) & u(k-1) & \ldots & u(k-h)\end{array}\right)^{T}$.

The relation (6) links on the time window the system's input and output to the initial state $x(k-h)$ of the system on the observation window.

We introduce the following proposition:

Assumption 1: The observability matrices $\mathcal{O}_{\mu, h}$ of the paths $\mu$ generated on the observation window $[k-h, k]$ are all of full rank: $\operatorname{rank}\left(\mathcal{O}_{\mu, h}\right)=\operatorname{dim}(x)=n, \forall h \geq n$.

The existence of an integer $h$, such that assumption 1 holds, has been analyzed in [10] and is linked to pathwise observability that have been furthermore shown to be decidable. Moreover, assumption 1 implies that all the pairs $\left(C, A_{\mu_{h}}\right)$ are observable for any $\mu_{h}, \mu_{h} \in \Theta_{h}$.

Using assumption 1 , one can define a projection matrix ${ }^{1} \Omega_{\mu, h}$ in such a way that $\Omega_{\mu, h} \mathcal{O}_{\mu, h}=0$, i.e. $\Omega_{\mu, h}$ is selected as a basis for the left null space of $\mathcal{O}_{\mu, h}$.

Next, residuals $r_{\mu, h}(\cdot)$, independent of the initial state $x(k-$ $h$ ), to be used for detection of the active path on the observation window can be defined as:

$$
r_{\mu, h}(k)=\Omega_{\mu, h}\left(Y_{k-h, k}-\mathcal{T}_{\mu, h} U_{k-h, k}\right)
$$

The $r_{\mu, h}(\cdot)$ residuals depends only on the system's input and output and their calculation requires the preliminary determination of all matrices $\Omega_{\mu, h}$.

Theorem 1 (Active path determination): The active path $\mu^{*}$ describing the true mode sequence on a time window $[k-h, k]$ satisfies:

$$
r_{\mu^{*}, h}(k)=\Omega_{\mu^{*}, h}\left(Y_{k-h, k}-\mathcal{T}_{\mu^{*}, h} U_{k-h, k}\right)=0
$$

To recover the true mode sequence $\mu^{*}$ from the system's measurements, one can proceed in the following way:

- first, all the possible paths of length $h$ are built on the time window $[k-h, k]$. This is equivalent to finding all the matrices $\mathcal{O}_{\mu, h}$.

${ }^{1}$ In fact, the existence of the projection matrix is linked to the observability of the system and to the length of the observation window [11]. 
- knowing the matrices $\mathcal{O}_{\mu, h}$, the projection matrices $\Omega_{\mu, h}$ are easily calculated.

- from the matrices $O_{\mu, h}$ and $\Omega_{\mu, h}$, one can form the residuals $r_{\mu, h}(\cdot)$ using the system's measurements.

- the active path is recovered from the system's measurements by testing the residuals $r_{\mu, h}(\cdot)$ magnitude and it corresponds to the one which residual is equal to zero.

\section{A. On the number of path}

It is easy to see that the enumeration of all paths on a time window $[k-h, k]$ introduces a problem of combinative explosion related to the number of modes and the length of the observation window. Indeed, the number of residuals $r_{\mu, h}(\cdot), \mu \in \Theta_{h}$, to be calculated is equal to $s^{h}$ and quickly grows with the length $h+1$ of the observation window and the number $s$ of modes. Then, the use of all paths on a time window is awkward and computationally demanding.

In practice, all paths $\mu \in \Theta_{h}$ do not have to be considered at every moment. When at a time $k_{0}$, the active path on an observation window $\left[k_{0}-h, k_{0}\right]$ is identified, it is not necessary to test the $s^{h}$ residuals at the next instant $k_{0}+1$. Only the paths $\mu \in \Theta_{h}$ with infixes $\mu_{\left[k_{0}+1-h, k_{0}-1\right]}$ identical to the infix $\mu_{\left[k_{0}+1-h, k_{0}-1\right]}^{*}$ of the previously recovered path $\mu^{*}$ at $k_{0}$ are considered at the next instant $k_{0}+1$. For example, from table I, one can see that if at time $k_{0}$, the recovered active path is the path $\mu^{*}=(1 \cdot 2 \cdot 1)$ (path $\mu^{2}$ ), then only the paths $\mu=(2 \cdot 1 \cdot 1)$ (path $\mu^{5}$ ) and $\mu=(2 \cdot 1 \cdot 2)$ (path $\mu^{7}$ ) have to be considered at the next step $k_{0}+1$.

Moreover, assuming that the minimum sojourn time in a mode is greater than the length of the observation window, one can limit the number of generated paths by only considering paths that describe the mode sequence when the system remains in the same mode all over the duration of the observation window, i.e. $\mu=(i \cdot i \cdot \ldots \cdot i), i \in\{1,2, \ldots, s\}$. In table I, the paths $\mu^{1}$ and $\mu^{8}$ describe the mode sequence when the system remains respectively in mode 1 and 2 all over the duration of the time window. Nevertheless, the reduction of the number of residuals comes at the expense of a delay in the estimation of the switching time from one mode to another. The recognition of the active path cannot take place as long as the switching instant is in the observation window. Thus, a maximum delay equals to the length of the observation window exists.

Prior knowledge on the process such as "prohibited" switching sequences or minimal time between two consecutive switchings, can also help to limit the number of generated residuals or paths to be considered.

\section{PATH DISCERNABILITY}

In what follows, we are interested in the conditions guaranteeing the discernability of the various paths enumerated on an observation window. These conditions ensure the uniqueness of the recovered active path $\mu^{*}$ during the path recognition process. Discernability guarantees that two different modes never induce the system in the same dynamics on a finite time window.
Definition 4 (Path discernability): Two paths $\mu^{1} \in \Theta_{h}$ and $\mu^{2} \in \Theta_{h}$ are discernible on an observation window $[k-h, k]$ if their respective corresponding residuals $r_{\mu^{1}, h}(\cdot)$ and $r_{\mu^{2}, h}(\cdot)$ are not simultaneously null when one of the two paths is active on the considered observation window.

In order to establish the discernability conditions of two different paths, let us consider two paths $\mu^{1} \in \Theta_{h}$ and $\mu^{2} \in \Theta_{h}$ on an observation window $[k-h, k]$. We denote $Y_{k-h, k}^{\mu^{1}}$ (respectively $Y_{k-h, k}^{\mu^{2}}$ ) the output vector related to the system when it undergoes the path $\mu^{1}$ (respectively $\mu^{2}$ ). We suppose that at an instant $k$, the active path on the observation window is the path $\mu^{1}$. This information being unknown, we have to analyze the possibilities that the path $\mu^{1}$ or the path $\mu^{2}$ are in adequacy with the system's data. From (7), the expressions of the residuals $r_{\mu^{1}, h}(\cdot)$ and $r_{\mu^{2}, h}(\cdot)$ are given by :

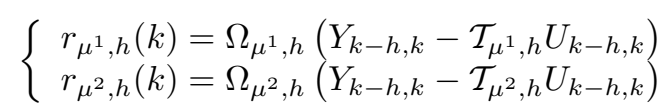

Since $\mu^{1}$ is assumed to be the active path on the observation window, and by definition $\Omega_{\mu^{1}, h}\left(Y_{k-h, k}^{\mu^{1}}-\mathcal{T}_{\mu^{1}, h} U_{k-h, k}\right)=0$, equation (9) can be written as:

$$
\left\{\begin{array}{l}
r_{\mu^{1}, h}(k)=0 \\
r_{\mu^{2}, h}(k)=\Omega_{\mu^{2}, h}\left(Y_{k-h, k}^{\mu^{1}}-\mathcal{T}_{\mu^{2}, h} U_{k-h, k}\right)
\end{array}\right.
$$

Adding and taking away $Y_{k-h, k}^{\mu^{2}}$ from the expression of $r_{\mu^{2}, h}(\cdot)$, one obtains:

$$
\left\{\begin{aligned}
r_{\mu^{1}, h}(k)= & 0 \\
r_{\mu^{2}, h}(k)= & \Omega_{\mu^{2}, h}\left(Y_{k-h, k}^{\mu^{1}}-Y_{k-h, k}^{\mu^{2}}\right. \\
& \left.+Y_{k-h, k}^{\mu^{2}}-\mathcal{T}_{\mu^{2}, h} U_{k-h, k}\right)
\end{aligned}\right.
$$

As by definition $\Omega_{\mu^{2}, h}\left(Y_{k-h, k}^{\mu^{2}}-\mathcal{T}_{\mu^{2}, h} U_{k-h, k}\right)=0$, one has:

$$
\left\{\begin{array}{l}
r_{\mu^{1}, h}(k)=0 \\
r_{\mu^{2}, h}(k)=\Omega_{\mu^{2}, h}\left(Y_{k-h, k}^{\mu^{1}}-Y_{k-h, k}^{\mu^{2}}\right)
\end{array}\right.
$$

Equation (12) clearly points out that the residual calculated for the path $\mu^{2}$ (nonactive path) directly depends on the difference between the system's outputs when the mode sequence evolves according to the two paths $\mu^{1}$ and $\mu^{2}$, the system being excited by the same inputs in both cases. If we do not want the residuals $r_{\mu^{1}, h}(k)$ and $r_{\mu^{2}, h}(k)$ to be simultaneously equal to zero, a necessary and sufficient condition is:

$$
Y_{k-h, k}^{\mu^{1}}-Y_{k-h, k}^{\mu^{2}} \notin \mathcal{N}_{r}\left(\Omega_{2}\right)
$$

where $\mathcal{N}_{r}$ stands for the operator "right null space".

Thus, the condition (13) has to be analyzed in order to deduce the discernability conditions of paths $\mu^{1}$ and $\mu^{2}$.

According to (4), one has:

$$
\begin{aligned}
Y_{k-h, k}^{\mu^{1}}-Y_{k-h, k}^{\mu^{2}}= & \left(\mathcal{O}_{\mu^{1}, h}-\mathcal{O}_{\mu^{2}, h}\right) x(k-h) \\
& +\left(\mathcal{T}_{\mu^{1}, h}-\mathcal{T}_{\mu^{2}, h}\right) U_{k-h, k}
\end{aligned}
$$


where $x(k-h)$ is the value of the system's state at the initial instant of the observation window.

One deduces from (14) after multiplication on the left by $\Omega_{\mu^{2}, h}$ :

$$
\begin{array}{r}
\Omega_{\mu^{2}, h}\left(Y_{k-h, k}^{\mu^{1}}-Y_{k-h, k}^{\mu^{2}}\right)=\Omega_{\mu^{2}, h} \mathcal{O}_{\mu^{1}, h} x(k-h) \\
+\Omega_{\mu^{2}, h}\left(\mathcal{T}_{\mu^{1}, h}-\mathcal{T}_{\mu^{2}, h}\right) U_{k-h, k}
\end{array}
$$

If $Y_{k-h, k}^{\mu^{1}}-Y_{k-h, k}^{\mu^{2}}$ belongs to the right null space of $\Omega_{2}$, one has:

$$
\Omega_{\mu^{2}, h} \mathcal{O}_{\mu^{1}, h} x(k-h)+\Omega_{\mu^{2}, h}\left(\mathcal{T}_{\mu^{1}, h}-\mathcal{T}_{\mu^{2}, h}\right) U_{k-h, k}=0
$$

The relation is satisfied "for almost every initial state" $x(k-h)$ if the following necessary and sufficient condition is satisfied:

$$
\left\{\begin{array}{c}
\Omega_{\mu^{2}, h} \mathcal{O}_{\mu^{1}, h}=0 \\
\Omega_{\mu^{2}, h}\left(\mathcal{T}_{\mu^{1}, h}-\mathcal{T}_{\mu^{2}, h}\right) U_{k-h, k}=0
\end{array}\right.
$$

Therefore, the paths $\mu^{1}$ and $\mu^{2}$ are not discernible on a time window $[k-h, k]$ if the relations (17) are satisfied.

Theorem 2 (Path discernability): Two paths $\mu^{1}$ and $\mu^{2}$ of a switching system are discernible on an observation window $[k-h, k]$, "for almost every initial state" $x(k-h)$, if:

$$
\Omega_{\mu^{i}, h} \mathcal{O}_{\mu^{j}, h} \neq 0, \quad i, j \in\{1,2\}, i \neq j
$$

or

$$
\Omega_{\mu^{i}, h}\left(\mathcal{T}_{\mu^{j}, h}-\mathcal{T}_{\mu^{i}, h}\right) U_{k-h, k} \neq 0 \quad i, j \in\{1,2\}, i \neq j
$$

where $U_{k-h, k}$ is the vector containing the system's input stacked on the observation window.

The proof of this theorem directly comes from the preceding remarks.

When the paths $\mu^{1}$ and $\mu^{2}$ are of the type $(i, i, \ldots, i)$, $i \in\{1,2, \ldots, s\}$, theorem 2 is equivalent to the modes discernability conditions formulated in [12].

Remark 1 (Dependency to the initial state): In theorem 2 , the expression "for almost every initial state" holds owing to the fact that the discernability of the paths cannot be ensured for any initial state $x(k-h)$. In fact, for certain particular values of $x(k-h)$, the relation (16) is always satisfied independently of the input sequence $U_{k-h, k}$. For example, in the situation where $\mathcal{O}_{\mu^{1}, h}$ has full rank, for $x(k-h)=\left(\mathcal{O}_{\mu^{1}, h}\right)^{\dagger}\left(\Phi-\left(\mathcal{T}_{\mu^{1}, h}-T_{\mu^{2}, h}\right) U_{k-h, k}\right)$, equation (16) is satisfied for every input sequence $U_{k, k-h}$, where $\Phi$ belongs to the right null space of $\Omega_{\mu^{2}, h}$ and $\left(\mathcal{O}_{\mu^{1}, h}\right)^{\dagger}$ is the pseudo-inverse of $\mathcal{O}_{\mu^{1}, h}$.

\section{DETERMINATION OF THE ACTIVE MODE IN NOISY ENVIRONMENT}

In section III, the determination of the active mode at any moment was carried out within a deterministic framework, i.e. there were no noise on the system's measurement. Now, we assume the presence of a bounded noise on the output

${ }^{2}$ see remark 1 for the explanation of the expression "for almost every initial state" of the system described by equation (1). The only available information on the noise is its maximum magnitude. No probabilistic assumption is formulated on the probability distribution of the measurement noise:

$$
\left\{\begin{array}{l}
x(k+1)=A_{\mu_{k}} x(k)+B u(k) \\
y(k)=C x(k)+n(k) \\
\forall k,|n(k)| \leq \delta, \quad \delta>0
\end{array}\right.
$$

where $\delta$ is the bound of measurement noise magnitude $n(\cdot)$. In this situation, the residual $r_{\mu^{*}, h}(\cdot)$, defined by (8) and which corresponds to the active path $\mu^{*}$ on the time window $[k-h, k]$, is no more equal to zero. Indeed, the expression of the residual $r_{\mu^{*}, h}(\cdot)$, using equation (7), becomes:

$$
r_{\mu^{*}, h}(k)=\Omega_{\mu^{*}, h}\left(Y_{k-h, k}-\mathcal{T}_{\mu^{*}, h} U_{k-h, k}+N_{k-h, k}\right)
$$

where the values taken by the measurement noise on the observation window $[k-h, k]$ are stacked in $N_{k-h, k}$. As $\Omega_{\mu^{*}, h}\left(Y_{k-h, k}-\mathcal{T}_{\mu^{*}, h} U_{k-h, k}\right)=0$, one can write :

$$
r_{\mu^{*}, h}(k)=\Omega_{\mu^{*}, h} N_{k-h, k}
$$

Using the bound of the measurement noise magnitude, we can define an interval residual $\left[r_{\mu^{*}, h}(k)\right]$ :

$$
\left[r_{\mu^{*}, h}(k)\right]=\left[\underline{r}_{\mu^{*}, h}, \bar{r}_{\mu^{*}, h}\right]
$$

where $\underline{r}_{\mu^{*}, h}$ and $\bar{r}_{\mu^{*}, h}$ depends on the bound $\delta$ of the measurement noise and are given by : $\underline{r}_{\mu^{*}, h}=-\left|\Omega_{\mu^{*}, h}\right| \mathbb{U} \delta$ and $\bar{r}_{\mu^{*}, h}=\left|\Omega_{\mu^{*}, h}\right| \mathbb{U} \delta, \mathbb{U}$ being a column vector of length equal to the number of column of $\Omega_{\mu^{*}, h}$ and all the elements of $\mathbb{U}$ being equal to 1 .

In an interval context, the determination of the active path amounts to seeking the path that corresponds to an interval residual including the value zero. This test can be performed by calculating the sign of the product of the upper and lower bounds of each interval residuals $\left[r_{\mu, h}(\cdot)\right]$. The interval residual $\left[r_{\mu, h}(\cdot)\right]$ associated with the active path $\mu^{*}$ is the one for which the sign of the product of its upper and lower bound is negative.

Depending on the evolution of the various operating regimes dynamics, it can happen that more than one interval residuals contains the value zero, this situation being linked to the path discernability and the bound of the measurement noise magnitude. In this case, one refrains from making any decision on the active path. We have to consider this situation from a looser point of view and we can only enumerate the set of all possible active paths.

\section{ACADEMIC EXAMPLE}

We present here an academic example of a switching system. The simulated system is characterized by three modes and the matrices of the models describing the different 
modes are:

$$
\begin{aligned}
& A_{1}=\left(\begin{array}{cc}
-0.211 & 0 \\
0 & 0.521
\end{array}\right) A_{2}=\left(\begin{array}{cc}
0.691 & 0 \\
0 & -0.310
\end{array}\right) \\
& A_{3}=\left(\begin{array}{cc}
0.153 & 0 \\
0 & 0.410
\end{array}\right) \\
& B=\left(\begin{array}{ll}
2 & -1
\end{array}\right)^{T} C=\left(\begin{array}{ll}
1 & 2
\end{array}\right)
\end{aligned}
$$

Figure 1 shows the input $u(\cdot)$, the output $y(\cdot)$, the state $x(\cdot)$ and the mode sequence $\mu_{(\cdot)}$. The vertical dashed lines on the third graphic of figure 1 mark the time instants at which switchings occur. The fourth graphic plots the mode sequence described by the mode selection variable $\mu_{(\cdot)}$. For instance, on the time windows $[1,8]$ and $[9,17]$, the system is respectively in the modes 1 and 2 .
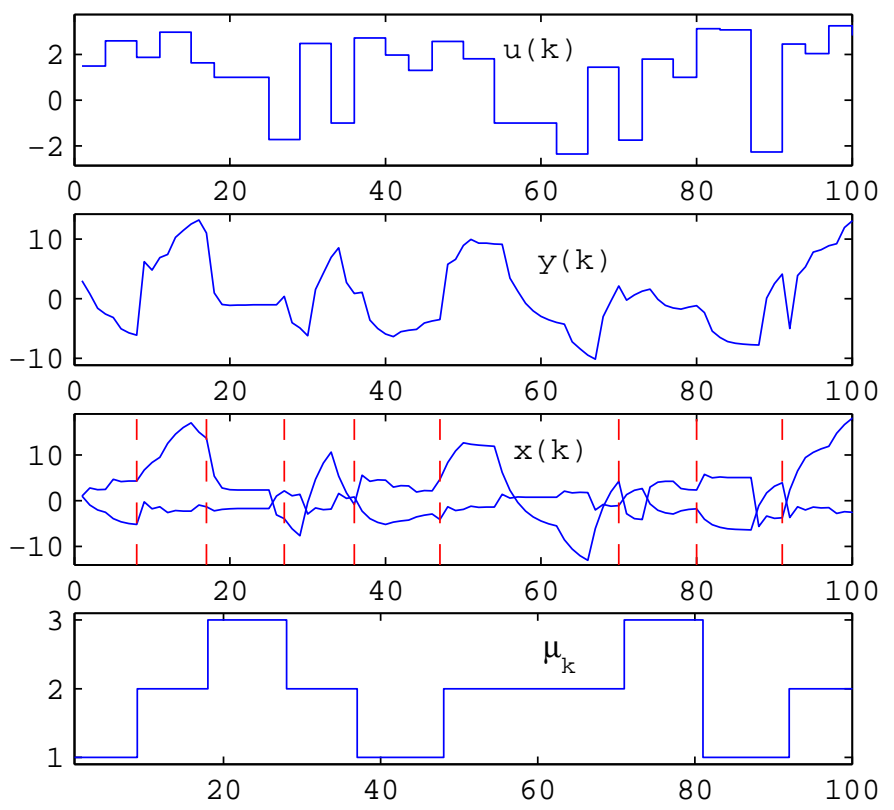

Fig. 1. Input $u(\cdot)$, output $y(\cdot)$, state $x(\cdot)$, mode sequence $\mu_{(\cdot)}$

As $\Omega_{l^{i}}^{k, h} O_{l^{j}}^{k, h} \neq 0, \mu^{i}, \mu^{j} \in \Theta_{2}, \mu^{i} \neq \mu^{j}, \Theta_{2}$ being the set of all paths of length 2 , the condition (18) of theorem 2 is respected. Condition (19) is tested at every moment. If it is not satisfied, no decision is taken concerning the recognition of the active path.

In order to perform the determination of the active path at every moment, we consider an observation window of length 3 . The set $\Theta_{2}$ of all paths of length 2 on the observation window corresponds to the set of the nine paths in table II.

TABLE II

SET OF ALL PATHS OF LENGTH 2

\begin{tabular}{lrrrrrrrrr}
\hline Path & $\mu^{1}$ & $\mu^{2}$ & $\mu^{3}$ & $\mu^{4}$ & $\mu^{5}$ & $\mu^{6}$ & $\mu^{7}$ & $\mu^{8}$ & $\mu^{9}$ \\
\hline$\mu_{1}$ & 1 & 1 & 1 & 2 & 2 & 2 & 3 & 3 & 3 \\
$\mu_{2}$ & 1 & 2 & 3 & 1 & 2 & 3 & 1 & 2 & 3 \\
\hline
\end{tabular}

As explained in section VI, in order to reduce the number of residuals to be analyzed during the mode's recognition process, one can consider only the paths describing the mode sequence when the system remains in the same mode all over the duration of the observation window. In this case, only the paths $(1 \cdot 1),(2 \cdot 2)$, and $(3 \cdot 3)$ have to be considered. The time evolution of the residuals $r_{(1 \cdot 1), h}(\cdot), r_{(2 \cdot 2), h}(\cdot)$ and $r_{(3 \cdot 3), h}(\cdot)$ corresponding to the three previous paths is illustrated on the graphics of figure 2. The graphics show that only one of the three residuals is null at every moment, except in a vicinity of the switching instants. The three residuals are not null in a vicinity of the switching instants because in this time window, none of the paths $(1 \cdot 1)$, $(2 \cdot 2)$ and $(3 \cdot 3)$ associated respectively with the three residuals $r_{(1 \cdot 1), h}(\cdot), r_{(2 \cdot 2), h}(\cdot)$ and $r_{(3 \cdot 3), h}(\cdot)$ matches the active path. These situations highlight the occurrence of a mode switching and one has to investigate other residuals associated to paths different from $(1 \cdot 1),(2 \cdot 2)$ and $(3 \cdot 3)$. The exploration of other residuals must be combined with the process of reduction of the number of paths as explained in section
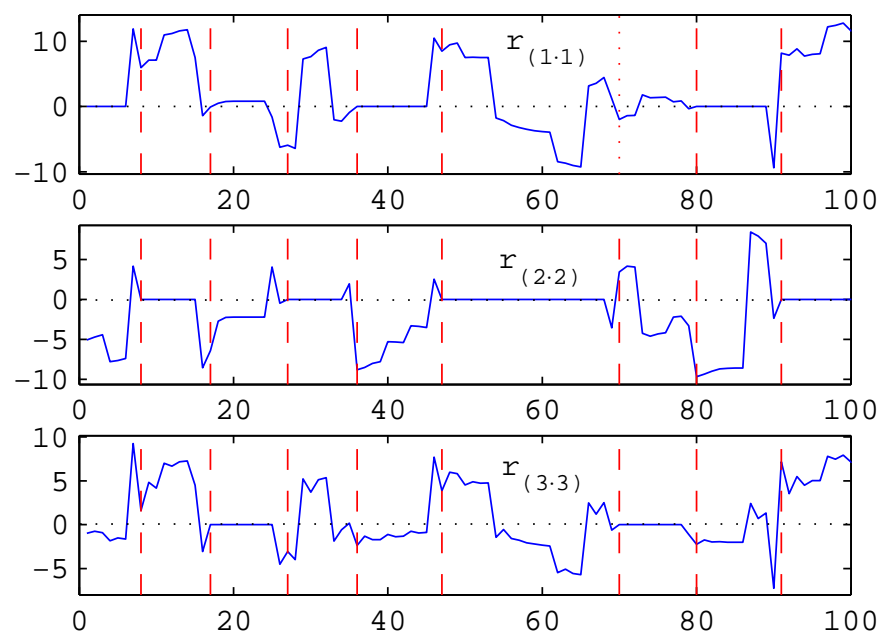

Fig. 2. Residuals $r_{(1 \cdot 1), h}(\cdot), r_{(2 \cdot 2), h}(\cdot)$ and $r_{(3 \cdot 3), h}(\cdot)$

The mode sequence (first graphic of figure 3) and its estimation (second graphic of figure 3) while analyzing the residuals are depicted on figure 3 . The figure shows that the mode sequence is exactly reconstructed.

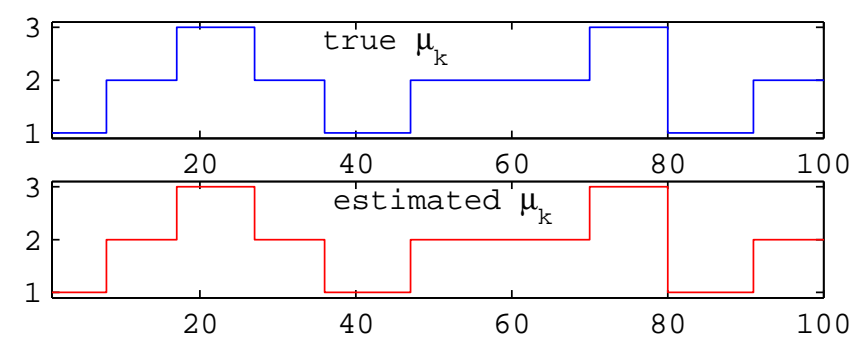

Fig. 3. Mode's recognition

The three graphics of figure 4 correspond to the obtained interval residuals in the case of the mode estimation in 
a noisy environment. The performed simulation takes into account the presence of a bounded measurement noise on the system's output with a magnitude that is at least equal to $10 \%$ of the magnitude of the system's ouput. The bounds of the interval residuals are represented on the three graphics of figure 4. Indeed, one can see that only one of the three interval residuals contains 0 at every moment, the index of this interval residual corresponding to the active path on the time window.
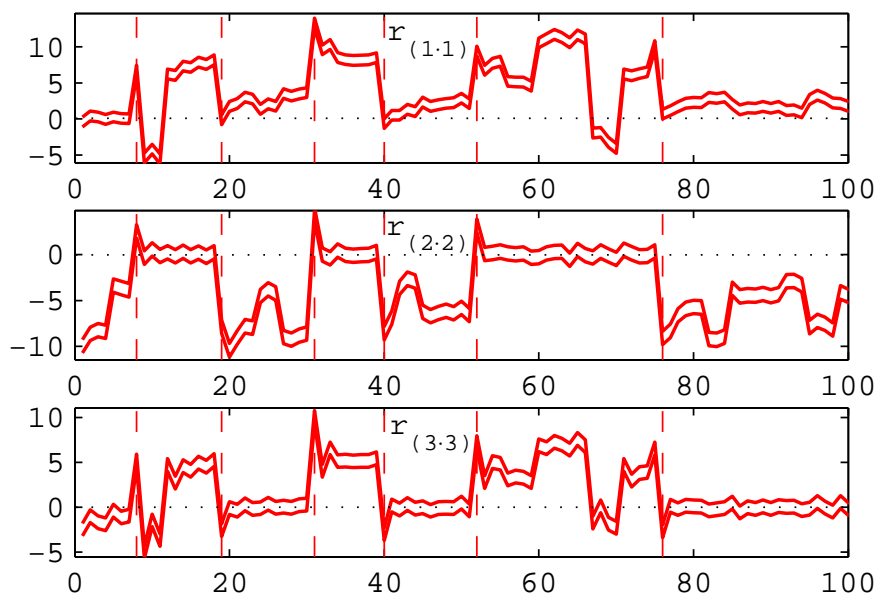

Fig. 4. Interval residuals

The results of the determination of the active mode are illustrated by figure 5. The first graphic of this figure indicates the simulated (true) mode sequence. The second graphic is obtained while analyzing the membership of the interval residuals regarding the value zero. Although the modes are rather well detected, there are situations where it is impossible to provide an estimate of $\mu_{(\cdot)}$ because more than one interval residual contains the value zero value or none of the three interval residuals contains the value zero. This kind of situation, due to the measurement noise and to the fact that all possible paths on the observation window are not considered in the analysis, is highlighted by points with ordinate equal to zero on the second graphic of figure 5. The third graphic of figure 5 is obtained while testing the coherence in the succession of the detected active paths at consecutive moments as indicated in section VI. One can noticed a perfect reconstruction of the mode sequence.

\section{CONCLUSION}

This paper proposed a method for the determination of the active mode and the switching instants of a switching system, using only the system's input and output data. We have also derived discernability conditions regardless of noise, i.e. conditions that ensure the uniqueness of the recovered mode.

It will be of great interest to consider in future work the situation where all the modes of the system are not previously indexed. In this case, one does not have a complete knowledge of all the operating regimes of the system. Therefore,

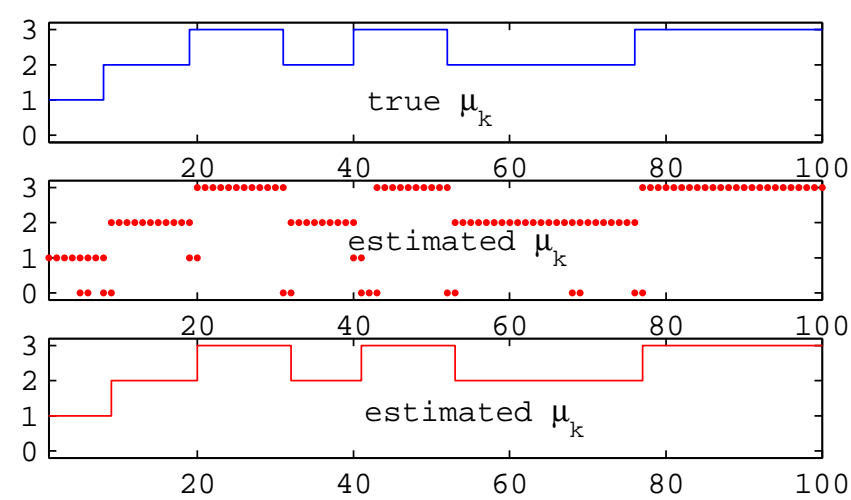

Fig. 5. Active mode recognition

it is necessary to simultaneously proceed to the detection of not indexed modes and the estimation of their parameters.

\section{REFERENCES}

[1] W. P. M. H. Heemels, B. De Schutter, and A. Bemporad, "Equivalence of hybrid dynamical models," Automatica, vol. 37(7), pp. 1085-1091, 2001.

[2] A. Juloski, M. Heemels, G. Ferrari-Trecate, R. Vidal, S. Paoletti, and H. Niessen, Hybrid Systems: Computation and Control, ser. Lecture Notes on Computer Science. Zurich, Switzerland: Springer-Verlag, 2005, vol. 3141, ch. Comparison of Four Procedures for Identification of Hybrid Systems.

[3] J. Ragot, G. Mourot, and D. Maquin, "Parameter estimation of switching piecewise linear systems," in Proceedings of the 42nd Conference on Decision and Control, Maui, Hawaii, 2003, pp. 5783-5788.

[4] R. Vidal, "Identification of PWARX hybrid models with unknow and possibly different orders," in Proceedings of the 23rd IEEE American Control Conference, Paradise Island, Bahamas, 2004, pp. 13-19.

[5] A. Bemporad, A. Garulli, S. Paoletti, and A. Vicino, "Data classification and parameter estimation for the identification of piecewise affine models," in Proceedings of the 43rd IEEE Conference on Decision and Control, Paradise Island, Bahamas, 2004, pp. 20-25.

[6] A. Bemporad and M. Morari, "Control of systems integrating logic, dynamics and constraints," Automatica, vol. 35(3), pp. 407-427, 1999.

[7] M. S. Branicky, V. S. Borkar, and S. K. Mitter, "A unified framework for hybrid control: model and optimal control theory," IEEE Transactions on Automatic Control, vol. 43(1), pp. 31-45, 1998.

[8] G. A. Ackerson and K. S. Fu, "On state estimation in switching environnements," IEEE Transactions on Automatic Control, vol. 15(1), pp. 10-17, 1970.

[9] A. Balluchi, L. Benvenuti, M. D. Di Benedetto, and A. L. SangiovanniVincentelli, "Design of observers for hybrid systems," in Proceedings of Hybrid Systems: Computation and Control, Berlin, Germany, 2002, pp. 76-89.

[10] M. Babaali, M. Egerstedt, and E. W. Kamen, "Pathwise observability and controllability are decidable," in Proceedings of the 42nd Conference on Decision and Control, Hawaii, USA, 2003, pp. 5771-5776.

[11] J. Gertler, Fault detection and diagnosis in engineering systems. Marcel Dekker, 1998.

[12] V. Cocquempot, M. Staroswiecki, and T. El Mezyani, "Switching time estimation and fault detection for hybrid systems using structured parity residuals," in Proceedings of the 15th IFAC Symposium on Fault Detection, Supervison and safety of Technical Processes, Washington, D. C., 2003, pp. 681-686. 